\title{
On General Measures of Deformation
}

\author{
By
}

\author{
R. L. Fosdick, Chicago, Ill., and A. S. Wineman, Ann Arbor, Mich. \\ (Received August 21, 1967)
}

Summary. Each particle of a continuum is assigned a second order tensor which is taken as a measure of the deformation of some neighborhood of the particle, and which is determined by a functional depending on the configurations of that neigh. borhood. Two invariance restrictions are imposed on the functional whose values are spatial strain tensors, that is, associated with the deformed configuration. The first requirement is that a time shift and rigid transformation of the deformed configuration leave the spatial deformation tensor unaltered relative to it. The second. requires that if particles of distinct continua undergo the same deformation, the corresponding deformation tensors should be the same. For the special case in which the functional depends on the deformation in the smallest neighborhood of a particle, the restrictions imply that the deformation tensors associated with the deformed and reference configurations are isotropic functions of the left and right CAUCHYGREEN tensors, respectively.

Zusammenfassung. Jedem Teilchen eines Kontinuums wird ein Tensor zweiter Stufe als Maß für die Deformation einer gewissen Nachbarschaft dieses Teilchen zugeordnet, der durch ein Funktional bestimmt wird, das von der Konfiguration dieser Nachbarschaft abhängt. Zwei Invarianzbedingungen werden diesem Funktional, dessen Werte räumliche Verzerrungstensoren darstellen, auferlegt, und zwar im Hinblick auf die deformierte Konfiguration. Die erste Forderung besagt, daß eine Zeitverschiebung und eine starre Transformation der deformierten Konfiguration den räumlichen Verzerrungstensor im Hinblick auf diese ungeändert lassen. Die zweite Einschränkung besagt, daß entsprechende Deformationstensoren von Partikeln verschiedener Kontinua, die dieselbe Verformung erlitten haben, gleich sein sollen. Im Spezialfall, daß die Funktionale nur von der Deformation in der nächsten Umgebung des Partikels abhängen, beinhalten die Einschränkungen die Aussage, daß die mit dem deformierten und dem undeformierten Zustand verknüpften Defor. mationstensoren nur isotrope Funktionen des linken und des rechten CAUCHYGreen Tensors sein können.

\section{Introduction}

The concept of strain as a measure of the change of local geometry of a deforming continuum has a long history dating back to the early seventeenth century ${ }^{1}$. Certain of the measures proposed since that time have met with general acceptance. These measures belong to a class whose general form satisfies a common set of underlying restrictive conditions which must logically be imposed upon any rational measure. The inadequacy of those measures which have not been found acceptable in general

\footnotetext{
I See Truespell and Toupin $[9], \S 33 \mathrm{~A}$ for an account of the history.
} 
studies of continuum mechanics has been due, we believe, essentially to an incomplete and insufficient understanding of these restrictive conditions. We remark that the acceptable measures have also been simple in the sense that only local geometric changes in vanishingly small neighborhoods of a particle are assumed to affect the strain at the particle. With the recent flourish of research on polar media it would appear that this restrictive assumption could stand generalization. This possibility of generalization combined with certain classical, as well as current day ${ }^{2}$ proposals for measures of strain. which are not properly invariant ${ }^{3}$, and which therefore, within the rational theory of continua, are not generally applicable, supplies the motivation for this investigation of general measures of deformation.

In order to develop a structure upon which all realistic measures of deformation should be built, we shall find it necessary to present those ideas which are most fundamental and essential to the concept of strain. Hence, after a brief section on kinematics, we begin in Section 3 with a general definition of strain at a particle in its motion, based on the intuitive feeling that it should depend upon the motion of the particles in some neighborhood of the fixed particle. Certain invariance restrictions which should be imposed on all realistic measures of strain are then discussed in Sections 4 and 5 . The results obtained are applied to the limiting case of simple strain in which the neighborhood of a particle, which is assumed to influence the value of strain at the particle, is taken vanishingly small. In particular, Section 4 contains a statement of the implications of our first postulate which says, roughly, that if two motions of a continuum differ only with respect to when they are initiated, and after being initiated are related by a continuous time parameter sequence of rigid body transformations, the strains should remain intrinsically unaltered ${ }^{4}$. In Section 5 we similarly treat our second postulate, which is essentially a requirement that if particles of materially distinct continua are subjected to intrinsically the same local kinematics then intrinsically the strain at these particles should be the same ${ }^{5}$. Strain measures which satisfy the two invariance postulates discussed in Sections 4 and 5 are said to be properly invariant.

In Section 6, we consider the special case of simple strain for which the invariance postulates yield a general canonical form. It is shown that the concept of simple strain is completely characterized by the necessary and sufficient condition that the strain rate tensor must vanish as a con-

${ }^{2}$ The most recent appears to be that of KARNI and REINER [7] in which they present two measures of strain, in addition to those commonly named after GREEN and Almaxsi, which are not properly invariant.

${ }^{3}$ In $\S \S 3,4$ of the present paper, we consider the question of properly invariant measures of strain.

4 This postulate is analogous to the principle of material frome indifference which is used in modern treatments of constitutive equations in continuum mechanics. See, e.g., Truesdell and Nolt [1].

${ }_{5}$ This postulate is analogous to the concepts of isotropy and homogeneity in continuum mechanics. Again see [1]. 
sequence of the vanishing of the deformation gradient rate tensor at a given particle. In other words the neighborhood which influences the strain at a particle must be arbitrarily small if the strain rate tensor is assumed to vanish whenever the deformation gradient rate tensor vanishes at the particle. The possibilities of first and second order simple strain measures are discussed. Aside from these later results, some of the more significant general results obtained in Sections 4, 5 and 6 are summarized at the end of Section 3.

Throughout most of this paper, our central considerations are on spatial measures of strain $^{6}$. In Section 7 , material strain measures are introduced and related to the spatial measures, obviating the need for a detailed independent treatment. Results for the special case of simple material measures of strain are then discussed.

Notation. The usual direct notation of matrix calculus will be employed; bold face capital letters denote second order tensors and bold face lower case letters denote vectors or vector fields. Exceptions to this rule are $\mathbf{X}_{r}, \mathbf{Y}_{r}, \widehat{\mathbf{Y}}_{\hat{q}}$, and various other subscripted versions of the base letters $\mathbf{X}$ and $\mathbf{Y}$. These denote position vectors. The symbols $f, g, h$ will designate tensor valued functions. We shall use $\mathbf{1}$ to denote the unit matrix and 0 to denote either the null vector or the null tensor. Matrix inversion, transpose, trace, and determinant are denoted respectively by $\mathbf{A}^{-1}, \mathbf{A}^{T}, \operatorname{tr} \mathbf{A}$, and $\operatorname{det} \mathbf{A}$. The gradient operator $\nabla$ denotes position gradient. The space-like independent variables with respect to which the gradient is intended will be stated explicitly as arguments of the function to which $\nabla$ is applied. For example, $\nabla \mathbf{b}(\mathbf{x})$, denotes the matrix of partial derivatives of $\mathbf{b}$ with respect to $\mathbf{x}$ while $\nabla \mathbf{b}(\mathbf{X})$ denotes the matrix of partial derivatives of $\mathbf{b}$ with respect to $\mathbf{X}$. Finally, light faced capital letters $X, Y, \widehat{X}, \widehat{Y}$ denote material particles, and $\mathfrak{R}$, $\hat{\mathfrak{R}}$, and various subscripted forms of these symbols denote neighborhoods.

\section{Kinematics}

The motion of a continuous medium or body is completely specified by an invertible functional relation between its particles $X$ and their positions in space $\mathbf{x}$ as time $t$ progresses;

$$
\mathrm{x}=\chi(X, t) \text {. }
$$

Equation (2.1) is rendered useful for the derivation of certain concepts in continuum mechanics by introducing a method of naming or labeling the particles. This is conveniently accomplished by a one-to-one mapping of the particles $X$ of the body into a region of three-dimensional EvcuidDean space called a reference configuration $\mathbf{r}$. Hence, we write

$$
\mathbf{X}_{r}=\mathbf{r}(X),
$$

which denotes the position $\mathbf{X}_{r}$ occupied by particle $X$ in reference configuration $\mathbf{r}$. We write the motion, referred to $\mathbf{r}$, as $^{7}$

$$
\mathbf{x}=\boldsymbol{\chi}\left(\mathbf{r}^{-1}\left(\mathbf{X}_{r}\right), t\right)=\chi\left(\mathbf{X}_{r}, t\right) .
$$

6 See Section 3 for this definition.

'No confusion should arise by using the same symbol $\chi$ to represent the motion both in the form (2.1) and in the form (2.3), referring it to $\mathbf{r}$. 
Several choices of reference configurations are presently used in continuum mechanics. A reference configuration which is often employed is one associated with some previous state (e.g. undeformed, if it exists) of the body. Although we do not use such an assumption here, it may be convenient to think of $\mathbf{r}$ in this manner.

Let $Y$ be a particle in some neighborhood $\mathfrak{R}(X)$ of $X$. The localization of the motion $\chi$ at $X$ in $\mathfrak{R}(X)$ is defined by

$$
\chi X(Y, t)=\chi(Y, t)-\chi(X, t), \quad Y \in \mathfrak{R}(X),
$$

and represents the motion of particle $Y$ relative to the motion of $X$. We observe that

$$
\chi_{X}(X, t)=0 .
$$

It will be convenient to express this localization in terms of the position of $Y$ relative to the position of $X$ in reference configuration $\mathbf{r}$. Toward this end, and analogous to (2.2) the position of $Y$ in $\mathbf{r}$ is given by

$$
\mathbf{Y}_{r}=\mathbf{r}(Y) \text {. }
$$

Hence the position of $Y$ relative to $X$ in $\mathbf{r}$ becomes

$$
\mathbf{r}_{X}(Y)=\mathbf{r}(Y)-\mathbf{r}(X)=\mathbf{Y}_{r}-\mathbf{X}_{r} .
$$

Employing the notation $\Delta \mathbf{Y}_{r}$ to denote this relative position vector in $\mathbf{r}$, we have

$$
\Delta \mathbf{Y}_{r}=\mathbf{r}_{X}(Y), \quad \Delta \mathbf{X}_{r}=\mathbf{r}_{X}(X)=\mathbf{0} .
$$

In the mapping (2.8) of $Y \rightarrow \Delta \mathbf{Y}_{r}$ for fixed $X$, the neighborhood $\mathfrak{R}(X)$ in reference configuration $\mathbf{r}$ is mapped into a neighborhood of the null vector 0 . We will denote this neighborhood by $\mathfrak{R}_{r}(\mathbf{0})$, and designate it as a neighborhood of the null vector in reference configuration r. Accordingly,

$$
Y \in \mathfrak{R}(X) \Rightarrow \lambda \mathbf{Y}_{r} \in \mathfrak{N}_{r}(\mathbf{0}) .
$$

Finally, by the inversion of (2.8), it follows from the localization (2.4) that

$$
\chi_{X}(Y, t)=\chi_{X}\left(\mathbf{r}_{X^{-1}}\left(\Delta \mathbf{Y}_{r}\right), t\right)=\chi_{X}\left(\Delta \mathbf{Y}_{r}, t\right),
$$

in which the neighborhoods of definition are related by (2.9). Since $Y=X$ corresponds to $\Delta \mathbf{Y}_{r}=\mathbf{0}$, we obtain, in view of (2.5)

$$
\chi_{X}(0, t)=0 .
$$

We shall refer to $\chi_{X}\left(\Delta \mathbf{Y}_{r}, t\right), \Delta \mathbf{Y}_{r} \in \mathfrak{N}_{r}(\mathbf{0})$, as the localization of the motion $\chi$ at $\mathbf{0}$ in $\mathfrak{R}_{r}(\mathbf{0})$. As in (2.3) we have used the same symbol $\chi \chi X$ to represent the localized motion at $X$ independently of whether it is expressed in terms of neighboring particles or in terms of the relative position vector of neighboring particles in reference configuration $\mathbf{r}$.

The deformation gradient $\mathbf{F}$ at $X$ in the motion (2.1) is defined relative to a particular reference configuration. For the reference configuration $\mathbf{r}$ it is given by the non-singular tensor

$$
\mathbf{F}\left(\mathbf{X}_{r}, t\right)=\nabla \boldsymbol{\chi}\left(\mathbf{X}_{r}, t\right) .
$$


It follows from (2.4), (2.6), (2.7), (2.8) and (2.10) that the deformation gradient may also be calculated from the localization through

$$
\mathbf{F}\left(\mathbf{X}_{r}, t\right)=\left.\nabla \chi_{X}\left(\Delta \mathbf{Y}_{r}, t\right)\right|_{\Delta \mathbf{Y}_{r}=\mathbf{0}}=\nabla \boldsymbol{\chi}_{X}(\mathbf{0}, t) .
$$

The displacement $\mathbf{u}$ of $X$ in the motion (2.1) is defined as the position vector of $X$ at time $t$ relative to its position in a reference configuration. For reference configuration $\mathbf{r}$ we can either consider $\mathbf{u}$ at $X$ as a function of $\left(\mathbf{X}_{r}, t\right)$ or as a function of $(\mathbf{x}, t)$ and with the aid of (2.3) write accordingly

$$
\begin{gathered}
\mathbf{u}\left(\mathbf{X}_{r}, t\right)=\chi\left(\mathbf{X}_{r}, t\right)-\mathbf{X}_{r}, \\
\mathbf{u}(\mathbf{x}, t)=\mathbf{x}-\chi^{-1}(\mathbf{x}, t) .
\end{gathered}
$$

Then, the displacement gradients at $X$ are defined through

and

$$
\mathbf{P}\left(\mathbf{X}_{r}, t\right)=\nabla \mathbf{u}\left(\mathbf{X}_{r}, t\right)
$$

$$
\mathbf{M}(\mathbf{x}, t)=\nabla \mathbf{u}(\mathbf{x}, t)
$$

while chain differentiation, together with (2.12) provides the relation

$$
\mathbf{P}=\mathbf{M} \mathbf{F} .
$$

Using (2.14) and (2.16) it follows that $\mathbf{P}$ and $\mathbf{F}$ are directly related through

$$
\mathbf{P}=\mathbf{F}-\mathbf{1},
$$

while with the aid of $(2.18)$ we similarly reach

$$
\mathbf{M}=\mathbf{1}-\mathbf{F}^{-1} .
$$

\section{Strain}

We consider the strain at a particle $X$ in a continuum as a kinematical concept which is defined intuitively so as to represent a measure of the change in geometry of a neighborhood of $X$ as a consequence of deformation. The strain at a given time is a quantity which will then be determined through the comparison of the neighborhood of the particle at that time with some standard neighborhood. Moreover, the strain measure should depend on the size of the neighborhood of $X$ in which the change of geometry is being considered. We call the neighborhood of a particle which determines, through its local deformation, the value of the strain, the neighborhood of influence.

Because of the directional nature of the local geometric changes, it seems most natural that the strain should be defined as a tensorial quantity. For convenience we shall define strain as a second order tensor; the generalization of our approach to higher order tensors should be clear from the context. Therefore, the strain tensor $\mathbf{D}$ at $(X, t)$ in the motion $\chi$, is supposed to be determined through the second order tensor valued functional relation

$$
\mathbf{D}(X, t)=\underline{f}_{t}(\chi(Y, t) ; X), \quad Y \in \Re(X),
$$


where to be deterministic, $\underline{f}_{t}$ must satisfy

provided

$$
\underline{f}_{t}\left(\chi^{(1)}(Y, t) ; X\right)=\underline{f}_{t}\left(\chi^{(2)}(Y, t) ; X\right),
$$

$$
\chi^{(1)}(Y, t)=\chi^{(2)}(Y, t) \text { for } Y \in \mathfrak{R}(X),
$$

while $\Re(X)$ represents the neighborhood of influence. $f_{t}$ is a tensor valued functional which assigns to each local deformation of $\overline{\bar{X}}$ in $\mathfrak{N}(X)$ the value of the strain at $X$, the form which may depend on $t$, and, in general, on the particular continuum under consideration.

The "relative" quality of strain is implicit in the fundamental equations (3.1) if we recall that particles are assigned (reference) positions in some particular reference configuration, which can be taken as a comparison configuration. This is made more explicit if, in addition to (3.2) and (3.3), we further qualify the definition of strain by the normalizing assumption which recognizes that when a continuum is subject to a rigid motion from a particular reference configuration for all time $t$ it is intrinsically undisturbed relative to this reference configuration and which therefore asserts that its strain is zero. We shall return to this requirement later in Section 6.

In the present formulation, it is to be noted that we allow explicit dependence on the particle $X$ in $f_{t}$, which admits the possibility that even though the motion of the neighborhoods of influence of two distinct particles may be the same at time $t$, the strain at these two particles could be different.

For definiteness, we shall consider $\mathbf{D}(X, t)$ as a spatial strain measure in the sense that in a fixed coordinate system its components are defined relative to the base vectors at the (current) position $\mathrm{x}$ of $X$ at time $t$. By way of two invariance restrictions, postulated for all realistic measures of strain, we shall show the following necessary propositions concerning the strain functional:

(1) The form of $f_{t}$ is independent of time; $f_{t}=f$.

(2) The strain functional $f$ is homogeneous and thus doss not depend explicitly on the particle $X$;

$$
\underline{f}(\boldsymbol{\chi}(Y, t) ; X)=\underline{\underline{f}}(\boldsymbol{\chi}(Y, t)), \quad Y \in \mathfrak{R}(X) .
$$

(3) The strain functional $\underline{\underline{f}}$ is form invariant under changes of continua.

(4) The strain at $X$ depends only on the localization of the motion at $X$;

$$
\underline{f}(\chi(Y, t))=\underline{f}\left(\chi_{X}(Y, t), \quad Y \in \Re(X) .\right.
$$

In addition to these particular conclusions, mathematical statements of the two invariance postulates, when reduced by conclusions (1)-(4) above will remain as fundamental restrictive conditions on the formation of any realistic strain measure in the class covered by (3.1). These con- 
ditions include the implication that the strain functional must be an isotropic functional of the localization.

In the limit as the neighborhood of influence $\mathfrak{R}(X)$ is taken arbitrarily small we call the resulting strain measure simple. For a simple strain measure the restrictions imposed by the invariance postulates lead to the following additional conclusions;

(5) The strain tensor $\mathbf{D}$ must be symmetric; $\mathbf{D}=\mathbf{D}^{T}$.

(6) The strain tensor D must be an isotropic tensor valued function of the left CAUCHY-GREen tensor'.

\section{Invariance Condition of Frame Indifference}

In this section we present the first invariance restriction which we believe all realistic measures of strain should be required to satisfy. This restriction is analogous to the principle of material frame indifference which TRUESDELL and Noll have discussed in [1].

Associated with the motion $\chi$ of a continuum, consider a motion $\chi^{\prime}$ which is equivalent to $\chi$ in the sense that the material is deformed in all respects concerning time changes of internal geometry the same as in the motion $\chi$. However, the motion $\chi^{\prime}$ can be initiated at a different time than the motion $\chi$ and can be such that it appears to a fixed observer different from the motion $\chi$ by at most a continzous time parameter sequence of rigid orientations. Hence,

$$
\chi^{\prime}\left(X, t^{\prime}\right)=\mathbf{Q}(t) \chi(X, t)+\mathbf{c}(t), \quad t^{\prime}=t-a,
$$

where $a$ is an arbitrary scalar representing a shift in time $t$, where $\mathbf{c}(t)$ is an arbitrary vector valued function of time representing rigid translation of the motion $\chi^{\prime}$ compared to the motion $\chi$, and where $\mathbf{Q}(t)$ represents an arbitrary time dependent proper orthogonal transformation which accounts for the rigid rotation of the motion $\chi^{\prime}$ relative to the motion $\chi$;

$$
\mathbf{Q}(t) \mathbf{Q}^{T}(t)=\mathbf{Q}^{T}(t) \mathbf{Q}(t)=\mathbf{1}, \quad \operatorname{det} \mathbf{Q}(t)=\mathbf{1} .
$$

Roughly, the position of $X$ in motion $\chi$ at time $t$, and the position of $X$ in motion $\chi^{\prime}$ at a time unit earlier differ by a rigid body transformation.

The strain at $\left(X, t^{\prime}\right)$ in the motion $\chi^{\prime}$ is given, analogous to $(3.1)$, by

$$
\mathbf{D}^{\prime}\left(X, t^{\prime}\right)=\underline{f}_{t^{\prime}}\left(\chi^{\prime}\left(Y, t^{\prime}\right) ; X\right), \quad Y \in \mathfrak{R}(X) .
$$

We propose the following postulate concerning its relation to the strain D $(X, t)$ in the motion $\chi$.

Postulate I. In the equivalent motions $\chi^{\prime}$ of (4.1) and $\chi$ of $(2.1)$ of the same continum the corresponding spatial strain tensors $\mathbf{D}^{\prime}$ and $\mathbf{D}$ are related by the tensor transformation law

$$
\mathbf{D}^{\prime}\left(X, t^{\prime}\right)=\mathbf{Q}(t) \mathbf{D}(X, t) \mathbf{Q}^{T}(t) .
$$

8 The left CAUChY-GreEN strain tensor is defined in (6.14). 
This postulate makes precise the general feeling that in two motions of a continuum which differ only in time of occurence and by orientations in space the respective strain fields should appear intrinsically indistinguishable. For this reason, Postulate I. could be considered a postulate of observer invariance.

As is apparent from (3.1), and (4.1)-(4.4), the foregoing postulate places restrictions on the form of the strain functional as well as on the manner in which the strain at $X$ can depend on the motion in $\mathfrak{R}(X)$. These restrictions are of the same form as those considered by TRUesDELL and NoLL $[1]^{9}$ in their discussion of stress-deformation constitutive equations. Therefore, we shall not include the details here, but only mention that in a series of three special choices for the quantities $\mathbf{Q}(t), a$, and $\mathbf{c}(t)$ consisting of first, $\mathbf{Q}(t)=\mathbf{1}, a=0, \mathbf{c}(t)=-\chi(X, t)$ at $X$ fixed, second, $\mathbf{Q}(t)=\mathbf{1}, a=t, \mathbf{c}(t)=0$, and last, $\mathbf{Q}(t)$ any proper orthogonal transform, $a=0$, $\mathbf{e}(t)=0$, it follows, respectively, that the strain at $X$ depends on the motion in $\mathfrak{R}(X)$ through its localization (2.4),

$$
\mathbf{D}(X, t)=f_{t}\left(\chi_{X}(Y, t) ; X\right), \quad Y \in \mathfrak{N}(X),
$$

the form of the strain functional $f_{t}$ is independent of time

$$
\mathbf{D}(X, t)=\underline{f}\left(\chi_{X}(Y, t) ; X\right), \quad Y \in \mathfrak{M}(X),
$$

and that the strain functional $\underline{\underline{f}}$ satisfies the invariance condition

$$
\stackrel{f}{=}\left(\mathbf{Q}(t) \chi_{X}(Y, t) ; X\right)=\mathbf{Q}(t) \underset{=}{f}\left(\chi_{X}(Y, t) ; X\right) \mathbf{Q}^{T}(t), \quad Y \in \mathfrak{N}(X),
$$

for arbitrary transformations $\mathbf{Q}(t)$ which meet (4.2). We observe that the condition of determinism stated in (3.2), (3.3) becomes

provided

$$
\stackrel{f}{=}\left(\chi X^{(1)}(Y, t) ; X\right)=\underline{\underline{f}}\left(\chi_{X^{(2)}}(Y, t) ; X\right),
$$

$$
\chi X^{(1)}(Y, t)=\chi X^{(2)}(Y, t) \text { for } Y \in \mathfrak{R}(X) .
$$

The above results are independent of the choice of reference configuration. If we introduce the reference configuration $r$, it is possible, and also convenient, to express the strain $\mathbf{D}(X, t)$ in terms of the localization of the motion at 0 in $\mathfrak{R}_{r}(0)$ as given in (2.10). Thus, (4.6) becomes

$$
\mathbf{D}(X, t)=\underline{\underline{f_{r}}}\left(\boldsymbol{\chi} X\left(\Delta \mathbf{Y}_{r}, t\right) ; X\right), \quad \Delta \mathbf{Y}_{r} \in \mathfrak{N}_{r}(\mathbf{0}),
$$

where the notation $\underline{f}_{r}$ indicates that the form of the strain functional may depend on the particular reference configuration chosen. (4.10) differs from (4.6) in that the functional now depends on the values of the localization in $\mathfrak{N}_{r}(\boldsymbol{0})$ rather than in the neighborhood of influence $\mathfrak{R}(X)$ as in (4.6). Since the domains of definition of the localization are related by (2.8), (2.9), we will call $\mathfrak{R}_{r}(0)$ the neighborhood of influence in reference configuration $\mathbf{r}$. In addition to $(4.10)$ there are equations analogous to

9 See $\$ 26$. Also see the earlier work of NoLI [2]. 
(4.8), (4.9) expressing the property of determinism which we shall not repeat here. The invariance condition (4.7) trivially becomes

$$
\begin{gathered}
f_{r}\left(\mathbf{Q}(t) \chi_{X}\left(\Delta \mathbf{Y}_{r}, t\right) ; X\right)=\mathbf{Q}(t) f_{r}\left(\chi_{X}\left(\Delta \mathbf{Y}_{r}, t\right) ; X\right) \mathbf{Q}^{T}(t), \\
\Delta \mathbf{Y}_{r} \in \mathfrak{R}_{r}(\mathbf{0}),
\end{gathered}
$$

for all $\mathbf{Q}(t)$ which satisfy (4.2).

In the special case where the neighborhood of influence $\mathfrak{R}(X)$ is vanishingly small, we obtain from (2.9) the result that $\mathfrak{R}_{r}(0)$ is also vanishingly small, and with the aid of (2.11) and (2.13), we reach

$$
\chi_{X}\left(\Delta \mathbf{Y}_{r}, t\right) \doteqdot\left[\nabla \chi_{X}(0, t)\right] \Delta \mathbf{Y}_{r}=\mathbf{F}\left(\mathbf{X}_{r}, t\right) \Delta \mathbf{Y}_{r}, \quad \Delta \mathbf{Y}_{r} \in \mathfrak{N}_{r}(\mathbf{0}) .
$$

In this case, (4.10) and (4.12) imply the simple strain measure

$$
\mathbf{D}(X, t)=\underline{\underline{f_{r}}}\left(\mathbf{F}\left(\mathbf{X}_{r}, t\right) ; X\right),
$$

while the invariance condition (4.11) becomes

$$
\underline{f_{r}}\left(\mathbf{Q}(t) \mathbf{F}\left(\mathbf{X}_{r}, t\right) ; X\right)=\mathbf{Q}(t) \underline{\underline{f_{r}}}\left(\mathbf{F}\left(\mathbf{X}_{r}, t\right) ; X\right) \mathbf{Q}^{T}(t),
$$

for all $\mathbf{Q}(t)$ which meet (4.2).

Postulate I has been fully exploited and its implications are exhibited in (4.10) and (4.11). In the case of a simple strain measure, where the neighborhood of influence of the localized motion at $X$ on the strain at $(X, t)$ is vanishingly small, the results of Postulate $I$ are given in (4.13) and (4.14). We remark that similar results can also be generated for the cases where the neighborhood $\mathfrak{R}(X)$ is considered large enough to include second and higher deformation gradients. These investigations could be relevant to obtaining measures of strain for multipolar continua.

\section{Invariance Condition on the Definition of Strain}

In order to describe our second postulate of invariance, it is convenient to refer motions to reference configurations. For reasons of comparison we introduce two reference configurations $\mathbf{r}$ and $\hat{\mathbf{r}}$ each of which may be used to locate either particles $X, Y, \ldots$, of one continuum $\mathbb{E}$ or particles $\hat{X}, \hat{Y}, \ldots$, of a second continuum $\widehat{\mathbb{E}}$. We remark that the two continua $\mathbb{C}$ and $\widehat{\mathbb{E}}$ could be the same, in which case we write $\mathbb{C}=\widehat{\mathbb{E}}$ and interpret $X, Y, \hat{X}, \hat{Y}, \ldots$, as different particles of this common material. When it is essential to do so, we shall explicitly distinguish the case $\mathfrak{C}=\widehat{\mathfrak{C}}$. Otherwise, in the following discussion, this particular case should not be considered in any way as being special.

Analogous to (2.2) we may write

$$
\mathbf{X}_{\widehat{r}}=\hat{\mathbf{r}}(X),
$$

where $\mathbf{X}_{\hat{r}}$ denotes the position of particle $X$ in reference configuration $\widehat{\mathbf{r}}$. We will have occasion to consider the position of the same particle in both reference configurations $\mathbf{r}$ and $\hat{\mathbf{r}}$. The position of a particle $\hat{X}$ in 
reference configuration $\mathbf{r}$, of $\hat{X}$ in $\hat{\mathbf{r}}$, of $Y$ in $\hat{\mathbf{r}}, \ldots$, etc., are given respectively by $\hat{\mathbf{X}}_{r}=\mathbf{r}(\hat{X}), \hat{\mathbf{X}}_{\hat{r}}=\widehat{\mathbf{r}}(\hat{X}), \hat{\mathbf{Y}}_{\hat{r}}=\hat{\mathbf{r}}(Y), \ldots$, etc. Hence, in the spirit of (2.7), the position of $\hat{Y}$ relative to $\hat{X}$ in $\hat{\mathbf{r}}$ is given by

$$
\hat{\mathbf{r}}_{\hat{X}}(\hat{Y})=\widehat{\mathbf{r}}(\hat{Y})-\hat{\mathbf{r}}(\hat{X})=\hat{\mathbf{Y}}_{\widehat{r}}-\hat{\mathbf{X}}_{\hat{r}} .
$$

Analogous to $(2.8)$, we shall use the notation $\Delta \hat{\mathbf{Y}}_{\hat{r}}$ to denote this relative position vector in $\hat{\mathbf{r}}$;

$$
\Delta \hat{\mathbf{Y}}_{\hat{r}}=\hat{\mathbf{r}}_{\widehat{X}}(\hat{Y}), \quad \hat{\mathbf{r}}_{\widehat{X}}(\hat{X})=\mathbf{0} .
$$

Denoting by $\hat{\mathfrak{X}}(\hat{X})$ a neighborhood of particle $\hat{X}$, it follows that

$$
\widehat{Y} \in \hat{\mathfrak{R}}(\hat{X}) \Rightarrow \Delta \widehat{\mathbf{Y}}_{\hat{r}} \in \widehat{\mathfrak{N}}_{r}(0),
$$

where $\hat{\mathfrak{R}}_{\hat{\mathrm{r}}}(0)$ is a neighborhood of the null vector in $\hat{\mathbf{r}}$, which corresponds to the image of $\hat{\mathfrak{R}}(\widehat{X})$ under the mapping (5.2) and (5.3).

In a similar fashion $\Delta \hat{\mathbf{Y}}_{r}$ will denote the position vector of $\hat{Y}$ relative to $\hat{X}$ in the reference configuration $\mathbf{r}$. Then, from $(2.8)$ and (2.9) we have

$$
\Delta \hat{\mathbf{Y}}_{r}=\mathrm{r}_{\widehat{X}}(\hat{Y}), \quad \mathbf{r}_{\widehat{X}}(\hat{X})=\mathbf{0},
$$

and

$$
\hat{Y} \in \hat{\mathfrak{R}}(\hat{X}) \Rightarrow \Delta \widehat{\mathbf{Y}}_{r} \in \widehat{\mathfrak{N}}_{r}(\mathbf{0}),
$$

where $\hat{\mathfrak{R}}_{r}(0)$ is a neighborhood of the null vector in $\mathbf{r}$ corresponding to the image of $\hat{\mathfrak{R}}(\hat{X})$ under the mapping (5.5).

The motion (2.1), when considered relative to $\hat{\mathrm{r}}$, will be denoted $\hat{\chi}$, so that similar to (2.3) we may with the aid of (5.1) write

$$
\mathbf{x}=\hat{\boldsymbol{\chi}}\left(\mathbf{X}_{\hat{r}}, t\right) .
$$

Hence, to the localization of the motion $\chi$ at $\hat{X}$ in $\hat{\mathfrak{R}}(\hat{X})$ we can associate through (5.3), (5.4) and in a manner analogous to (2.10), the localization of the motion $\hat{\chi}$ at 0 in $\hat{\mathfrak{N}}_{\hat{r}}(0)$;

$$
\chi_{\hat{X}}(\hat{Y}, t)=\hat{\chi}_{\hat{X}}\left(\Delta \hat{\mathbf{Y}}_{\hat{r}}, t\right)
$$

where $\hat{Y}=\hat{X}$ corresponds, through (5.3), to $\Delta \hat{\mathbf{Y}}_{\hat{r}}=\mathbf{0}$, which implies

$$
\hat{\mathbf{\chi}}_{\widehat{x}}(\mathbf{0}, t)=\mathbf{0} \text {. }
$$

Similarly, we can also associate through (5.5), (5.6) the localization of the motion $\chi$ at 0 in $\hat{\Re}_{r}(0)$;

$$
\chi_{\widehat{X}}(\hat{Y}, t)=\chi_{\widehat{X}}\left(\Delta \hat{\mathbf{Y}}_{r}, t\right),
$$

where $\hat{Y}=\hat{X}$ corresponds, through (5.5), to $\Delta \hat{\mathbf{Y}}_{r}=\mathbf{0}$, which implies

$$
\boldsymbol{\chi x}_{\hat{x}}(\mathbf{0}, t)=\mathbf{0} .
$$


It is now straightforward to express the strain at $(\hat{X}, t)$ in terms of the localization of the motion at 0 in $\hat{\Re}_{r}(0)$ using (5.10), (5.6) and (4.6) rewritten in terms of $\hat{X}$ and $\hat{Y} \in \hat{\mathfrak{R}}(\hat{X})$ in just the same manner as (4.10) was expressed using (2.10), (2.9) and (4.6). Hence in the special case $\mathfrak{C}=\widehat{\mathbb{C}}$ we have

$$
\mathbf{D}(\hat{X}, t)=\underline{\underline{f}}_{r}\left(\boldsymbol{\chi} \hat{x}\left(\Delta \hat{\mathbf{Y}}_{r}, t\right) ; \hat{X}\right), \quad \Delta \hat{\mathbf{Y}}_{r} \in \hat{\mathfrak{R}}_{r}(0),
$$

whereas if the two continua are not the same, $\mathbb{E} \neq \widehat{\mathbb{E}}$, and the functional $\underline{\underline{f_{r}}}$ should be replaced by a different functional $\underline{\underline{f_{r}}}$ distinctive of the continuum $\widehat{\mathfrak{e}}$. In this development, if we replace the localization (5.10), (5.6) by the localization (5.8), (5.4) at 0 in $\hat{\mathfrak{R}}_{\hat{r}}(0)$, then we have essentially introduced the reference configuration $\widehat{\mathbf{r}}$, and, provided $\mathfrak{E}=\widehat{\mathbb{E}}$, the strain at $(\hat{X}, t)$ takes the form

$$
\mathbf{D}(\hat{X}, t)=\underline{\underline{f_{\hat{r}}}}\left(\hat{\boldsymbol{\chi}}_{\widehat{X}}\left(\Delta \hat{\mathbf{Y}}_{\hat{r}}, t\right) ; \hat{X}\right), \quad \Delta \hat{\mathbf{Y}}_{\hat{r}} \in \hat{\mathfrak{N}}_{\hat{r}}(\mathbf{0}),
$$

with again $f_{\hat{r}}$ being replaced by $\hat{f}_{\hat{r}}$ for the continuum $\widehat{\mathbb{C}}$ when $\mathbb{C} \neq \widehat{\mathbb{C}}$. Since (5.12) and (5.13) represent the strain at the same particle $\hat{X}$ in the same continuum at time $t$, we obtain a general relation between the strain functionals ${ }^{10} \underline{\underline{f}}_{r}$ and $\underline{\underline{f_{\hat{r}}}}$;

$$
\underline{\underline{f_{r}}}\left(\chi \widehat{X} \hat{x}\left(\Delta \hat{\mathbf{Y}}_{r}, t\right) ; \hat{X}\right)=\underline{\underline{f}} \hat{r}\left(\hat{\chi}_{\hat{X}}\left(\Delta \hat{\mathbf{Y}}_{\hat{r}}, t\right) ; \hat{X}\right),
$$

provided the localizations (5.8) and (5.10) of the motion $\chi$ associated with these reference configurations satisfy

$$
\chi_{\widehat{X}}\left(\Delta \hat{\mathbf{Y}}_{r}, t\right)=\hat{\chi}_{\widehat{X}}\left(\Delta \hat{\mathbf{Y}}_{\widehat{r}}, t\right)
$$

where, from $(5.3)-(5.6)$,

$$
\Delta \hat{\mathbf{Y}}_{\hat{r}}=\hat{\mathbf{r}}_{\widehat{X}}\left(\mathbf{r}_{\widehat{X}}^{-1}\left(\Delta \hat{\mathbf{Y}}_{r}\right)\right) \equiv \mathbf{h}_{\widehat{X}}\left(\Delta \hat{\mathbf{Y}}_{r}\right),
$$

with

$$
\Delta \hat{\mathbf{Y}}_{r} \in \widehat{\mathfrak{R}}_{r}(\mathbf{0}) \Rightarrow \Delta \hat{\mathbf{Y}}_{\hat{r}} \in \widehat{\mathfrak{R}}_{\hat{r}}(0) .
$$

It is through the mapping (5.16) that we may consider the neighborhood of influence $\widehat{\mathfrak{A}}_{\widehat{x}}(0)$ in $\widehat{\mathbf{r}}$ to be the image of the neighborhood of influence $\widehat{\Re}_{r}(0)$ in $\mathbf{r}$.

With a view toward a statement of our second invariance postulate, we now assume that a common reference configuration $\mathbf{r}$ of continua (s) and $\widehat{\mathbb{C}}$ is given and consider a special choice of a second reference con-

${ }^{10} \mathrm{It}$ is understood that if the two continua $\mathbb{E}$ and $\widehat{\mathbb{C}}$ are distinct then $f_{r}$ and $\underline{f_{r}}$ are to be replaced in (5.14) by the functionals $\underline{\underline{\hat{f}_{r}}}$ and $\underline{\underline{f_{\hat{r}}}}$ respectively, which are appropriate to the continuum $\hat{\mathbb{E}}$. 
figuration $\widehat{\mathbf{r}}$ for $\widehat{\mathfrak{C}}$. Let $X$ and $\hat{X}$ denote two arbitrarily chosen, but fixed, particles of continua $\mathbb{C}$ and $\widehat{\mathbb{C}}$ respectively, and associate with each particle $Y \in \mathfrak{R}(X)$ a particle $\hat{Y} \in \hat{\mathfrak{R}}(\hat{X})$ such that the distances between $X$ and $Y$ of $\mathfrak{C}$, and $\hat{X}$ and $\hat{Y}$ of $\hat{E}$ in the reference configuration $\mathbf{r}$ are equal;

$$
\left|\Delta \mathbf{Y}_{r}\right|=\left|\Delta \hat{\mathbf{Y}}_{r}\right| \text {. }
$$

Hence, the mapping of $\Delta \mathbf{Y}_{r} \in \mathfrak{R}_{r}(\mathbf{0}) \rightarrow \Delta \widehat{\mathbf{Y}}_{r} \in \hat{\mathfrak{R}}_{r}(\mathbf{0})$ is a rigid rotation with possible reflection

$$
\Delta \hat{\mathbf{Y}}_{r}=\mathbf{H} \Delta \mathbf{Y}_{r}, \quad \mathbf{H} \mathbf{H}^{T}=\mathbf{H}^{T} \mathbf{H}=\mathbf{1} .
$$

From the above, it follows that the neighborhoods $\mathfrak{N}_{r}(\mathbf{0})$ and $\hat{\mathfrak{N}}_{r}(\mathbf{0})$ of the null vector in reference configuration. $\mathrm{r}$ induced by (2.9) and (5.6) are congruent.

We now choose a second reference configuration $\widehat{\mathbf{r}}$ for $\widehat{\hat{C}}$ in such a way that the positions of its particles $\hat{X}$ and $\hat{Y}$ in this reference configuration coincide respectively with the positions of particles $X$ and $Y$ of $\mathbb{C}$ in $\mathbf{r}$. Then

$$
\hat{\mathbf{X}}_{\hat{r}}=\mathbf{X}_{r}, \quad \hat{\mathbf{Y}}_{\hat{r}}=\mathbf{Y}_{r},
$$

which, by subtracting the two equations, yields

$$
\Delta \hat{\mathbf{Y}}_{\hat{r}}=\Delta \mathbf{Y}_{r}
$$

with the implication, through (2.9) and (5.4), that

$$
\widehat{\mathfrak{N}}_{\widehat{r}}(\mathbf{0})=\mathfrak{R}_{r}(\mathbf{0}) .
$$

Thus, $\hat{\mathbf{r}}$ is related to $\mathbf{r}$ through a rigid transformation with possible rereflection which equivalently carries a neighborhood of $\hat{X}$, for $\widehat{\mathbb{E}}$ in $\mathbf{r}$, into coincidence with a neighborhood of $X$, for $\mathbb{C}$ in $\mathbf{r}$.

From (5.19) and (5.21) it follows that

$$
\Delta \hat{\mathbf{Y}}_{r}=\mathbf{H} \Delta \hat{\mathbf{Y}}_{\hat{r}} .
$$

This equation relates the relative positions of particles $\hat{X}$ and $\hat{Y}$ of $\widehat{\mathbb{C}}$ in the two reference configurations $\mathbf{r}$ and $\hat{\mathbf{r}}$, and, by inversion, represents a special choice of the function $\mathbf{h}_{\widehat{X}}$ in the mapping (5.16),

$$
\Delta \hat{\mathbf{Y}}_{\hat{r}}=\mathbf{H}^{T} \Delta \hat{\mathbf{Y}}_{r} .
$$

With the aid of (5.21) and (5.13), we may now express the strain at $(\hat{X}, t)$ for $\mathfrak{C}=\hat{\mathbb{C}}$ as

$$
\mathbf{D}(\hat{X}, t)=f_{\hat{r}}\left(\hat{\chi}_{\hat{X}}\left(\Delta \mathbf{Y}_{r}, t\right) ; \hat{X}\right), \quad \Delta \mathbf{Y}_{r} \in \mathfrak{R}_{r}(\mathbf{0}),
$$

where the functional $f_{\hat{r}}$ is to be replaced by $\hat{f}_{\hat{r}}$ if the two continua $\mathbb{E}$ and $\widehat{\hat{E}}$ are distinct. This brings us to the following 
Postulate II. If the reference configuration $\hat{\mathbf{r}}$ of $\widehat{\mathscr{C}}$ is chosen relative to the reference configuration $\mathbf{r}$ of $\mathbb{E}$ and $\widehat{\mathbb{C}}$ as implied in $(5.18)-(5.20)$, then the strain at $(X, t)$ in $\mathbb{C}$ due to the localization of a motion at $\mathbf{0}$ in $\mathfrak{R}_{r}(\mathbf{0})$, and the strain at $(\hat{X}, t)$ in $\widehat{\mathbb{S}}$ due to the same localization at $\mathbf{0}$ in $\hat{\mathfrak{S}}_{\hat{r}}(\mathbf{0})$, are equal.

Roughly, this postulate expresses the feeling that the strain at a particle should depend on its local kinematics in some neighborhood of influence, and, in addition, that if the neighborhoods of any two particles in materially distinct continua coincide in their reference configurations and also at time $t$, then the strain at time $t$ of these two particles should be the same.

A mathematical statement of this postulate can be constructed from (4.10) and (5.25) and reads for arbitrary $\mathbb{C}$ and $\widehat{\mathbb{C}}$,

$$
\underline{\underline{f_{r}}}\left(\boldsymbol{\chi} X\left(\Delta \mathbf{Y}_{r}, t\right) ; X\right)=\underline{\underline{f}} \hat{\underline{r}}\left(\hat{\mathbf{\chi}}_{X}\left(\Delta \mathbf{Y}_{r}, t\right) ; \hat{X}\right), \quad \Delta \mathbf{Y}_{r} \in \mathfrak{N}_{r}(\mathbf{0}),
$$
provided

$$
\chi_{X}=\hat{\chi}_{\hat{X}}=\bar{\chi}
$$

where $\bar{\chi}$ denotes an arbitrary function, and

A first consequence of Postulate II is the proposition that the strain functional of a continuum does not depend explicitly on the particle ${ }^{11}$,

$$
\underline{\underline{f_{r}}}\left(\boldsymbol{\chi}_{X}\left(\Delta \mathbf{Y}_{r}, t\right) ; X\right)=\underline{\underline{f_{r}}}\left(\boldsymbol{\chi}_{X}\left(\Delta \mathbf{Y}_{r}, t\right)\right) \text {. }
$$

To see this we need only consider in (5.19) the identity transformation $\mathbf{H}=1$. Then (5.21), (5.23) yield $\Delta \hat{\mathbf{Y}}_{r}=\Delta \hat{\mathbf{Y}}_{\hat{r}}=\Delta \mathbf{Y}_{r}$. This together with (5.14), (5.15), and letting $\chi_{X}=\hat{\chi}_{X}=\bar{\chi}$, implies for arbitrary $\mathfrak{C}$ and $\widehat{\mathbb{C}}$

$$
\underline{\underline{f_{r}}}\left(\overline{\mathbf{\chi}}\left(\Delta \mathbf{Y}_{r}, t\right) ; \hat{X}\right)=\underline{\underline{\hat{f}_{\hat{r}}}}\left(\overline{\mathbf{\chi}}\left(\Delta \mathbf{Y}_{r}, t\right) ; \hat{X}\right), \quad \Delta \mathbf{Y}_{r} \in \mathfrak{N}_{r}(\mathbf{0}),
$$

for arbitrary $\bar{\chi}$. We remark that in the case $\mathfrak{\mathbb { C }}=\hat{\mathfrak{E}}, \hat{f_{r}}$ and $\hat{f}_{\hat{r}}$ are replaced by the functionals $f_{r}$ and $\underline{f}_{\hat{r}}$ respectively. Hence, with the special assumption $\mathfrak{C}=\widehat{\mathfrak{C}},(5.26)$ and $(5.27 \mathrm{a})$ imply

$$
\underline{\underline{f_{r}}}\left(\overline{\mathbf{\chi}}\left(\Delta \mathbf{Y}_{r}, t\right) ; X\right)=\underline{\underline{f_{\hat{r}}}}\left(\bar{\chi}\left(\Delta \mathbf{Y}_{r}, t\right) ; \hat{X}\right), \quad \Delta \mathbf{Y}_{r} \in \mathfrak{N}_{r}(\mathbf{0}),
$$

and (5.29) and (5.30) yield

$$
\underline{\underline{f_{r}}}\left(\bar{\chi}\left(\Delta \mathbf{Y}_{r}, t\right) ; \hat{X}\right)=\underline{\underline{f_{r}}}\left(\overline{\bar{\chi}}\left(\Delta \mathbf{Y}_{r}, t\right) ; X\right), \quad \Delta \mathbf{Y}_{r} \in \mathfrak{N}_{r}(0),
$$

whatever the functional form of $\bar{\chi}$. Since this states that $f_{r}$ is independent of $X,(5.28)$ follows.

${ }^{11}$ An equivalent statement is that the strain functional is said to be homogeneous. 
A further consequence of Postulate II follows if we take account of (5.27a) and reduce (5.26) and (5.29) by means of the proposition above to the respective forms

and

$$
\underline{f}_{r}\left(\bar{\chi}\left(\Delta \mathbf{Y}_{r}, t\right)\right)=\underline{\underline{f}}_{\vec{r}}\left(\overline{\mathbf{\chi}}\left(\Delta \mathbf{Y}_{r}, t\right)\right)
$$

$$
\underline{\underline{\hat{f}_{r}}}\left(\overline{\mathbf{X}}\left(\Delta \mathbf{Y}_{r}, t\right)\right)=\underline{\underline{f}}_{\hat{r}}\left(\overline{\mathbf{\chi}}\left(\Delta \mathbf{Y}_{r}, t\right)\right)
$$

for $\Delta \mathbf{Y}_{r} \in \mathfrak{N}_{r}(0)$, and for arbitrary functions $\bar{\chi}$. Hence, equating the left hand sides above we arrive at the proposition that strain functionals are form invariant under changes of material,

$$
\underline{\underline{f}}=\underline{\underline{f}} .
$$

We note that it follows from the first of (5.32) together with (5.33) that

$$
\underline{\underline{f}}_{r}\left(\overline{\boldsymbol{\chi}}\left(\Delta \mathbf{Y}_{r}, t\right)\right)=\underline{\underline{f_{r}}}\left(\overline{\boldsymbol{\chi}}\left(\Delta \mathbf{Y}_{r}, t\right)\right), \quad \Delta \mathbf{Y}_{r} \in \mathfrak{N}_{r}(\mathbf{0}),
$$

for arbitrary $\bar{\chi}$.

Now, recall the condition $(5.27 \mathrm{~b})$ of Postulate II which requires that $\mathbf{r}$ and $\widehat{\mathbf{r}}$ be so related that the relation (5.16) between $\Delta \hat{\mathbf{Y}}_{r}$ and $\Delta \widehat{\mathbf{Y}}_{\widehat{r}}$ has the special form (5.24). Then (5.14), reexpressed using (5.34), the first consequence of Postulate II, and (5.15) supplemented by (5.24), yields the general invariance restriction ${ }^{12}$

$$
\underline{\underline{f_{r}}}\left[\chi_{\widehat{X}}\left(\Delta \hat{\mathbf{Y}}_{r}, t\right)\right]=\underline{\underline{f}}_{\underline{r}}\left[\hat{\mathbf{\chi}}_{\widehat{X}}\left(\Delta \hat{\mathbf{Y}}_{\hat{r}}, t\right)\right]
$$

provided

$$
\chi_{\hat{x}}\left(\Delta \hat{\mathbf{Y}}_{r}, t\right)=\hat{\chi}_{\hat{x}}\left(\Delta \hat{\mathbf{Y}}_{\hat{r}}, t\right),
$$

and

$$
\Delta \hat{\mathbf{Y}}_{\hat{r}}=\mathbf{H}^{T} \Delta \hat{\mathbf{Y}}_{r}, \quad \Delta \widehat{\mathbf{Y}}_{r} \in \widehat{\mathfrak{A}}_{r}(\mathbf{0}),
$$

for arbitrary constant orthogonal transformations $\mathbf{H}$.

In the special case where the neighborhood of influence $\mathfrak{M}(X)$ is vanishingly small, then, as remarked earlier, (2.9) implies that $\mathfrak{N}_{r}(0)$ is vanishingly small. This, in turn, through (5.22), also yields $\hat{\mathfrak{R}}_{\hat{r}}(0)$ vanishingly small. Thus, through $(5.23),(5.4)$ and $(5.6)$ we also have $\widehat{\mathfrak{N}}_{r}(0)$ vanishingly small. With reference to $(5.9)$ and $(5.11)$, these remarks imply that in addition to the approximation (4.12), we also have

$$
\begin{array}{ll}
\mathbf{\chi} \widehat{x}\left(\Delta \hat{\mathbf{Y}}_{r}, t\right) \doteq\left[\nabla \chi_{\widehat{x}}(\mathbf{0}, t)\right] \Delta \hat{\mathbf{Y}}_{r}=\mathbf{F}\left(\hat{\mathbf{X}}_{r}, t\right) \Delta \hat{\mathbf{Y}}_{r}, & \Delta \hat{\mathbf{Y}}_{r} \in \hat{\mathfrak{N}}_{r}(\mathbf{0}), \\
\hat{\mathbf{X}}_{\hat{x}}\left(\Delta \hat{\mathbf{Y}}_{\hat{r}}, t\right) \doteq\left[\nabla \hat{\mathbf{X}}_{\hat{x}}(0, t)\right] \Delta \hat{\mathbf{Y}}_{\hat{r}}=\hat{\mathbf{F}}\left(\hat{\mathbf{X}}_{\hat{r}}, t\right) \Delta \hat{\mathbf{Y}}_{\hat{r}}, \quad \Delta \hat{\mathbf{Y}}_{\hat{r}} \in \hat{\mathfrak{N}}_{\hat{r}}(\mathbf{0}),
\end{array}
$$

where

$$
\mathbf{F}\left(\hat{\mathbf{X}}_{r}, t\right)=\nabla \chi\left(\hat{\mathbf{X}}_{r}, t\right)=\nabla \chi_{\hat{X}}(\mathbf{0}, t),
$$

12 A less precise equivalent statement of this result is that the strain functional is said to be isotropic. 


$$
\hat{\mathbf{F}}\left(\hat{\mathbf{X}}_{\hat{r}}, t\right)=\nabla \hat{\mathbf{\chi}}\left(\hat{\mathbf{X}}_{\widehat{r}}, t\right)=\nabla \hat{\mathbf{\chi}}_{\widehat{X}}(\mathbf{0}, t),
$$

are deformation gradients of the motions $\chi$ and $\hat{\chi}$, respectively. The equivalence of the deformation gradients to the gradients of the localizations as stated in (5.38) and (5.39) follow in the same manner as in the analogous situation (2.13). The approximations (5.36) and (5.37) imply that (5.12) and (5.13) reduce to simple strain measures, depending only on the deformation gradients, analogous to the manner in which (4.10) reduced to $(4.13)$. In terms of these simple strain measures, $(5.35 \mathrm{a}-\mathrm{c})$, reduce to

where

$$
\underline{\underline{f}}_{r}\left[\mathbf{F}\left(\hat{\mathbf{X}}_{r}, t\right)\right]=\underline{\underline{f_{r}}}\left[\hat{\mathbf{F}}\left(\hat{\mathbf{X}}_{\hat{r}}, t\right)\right],
$$

$$
\mathbf{F}\left(\hat{\mathbf{X}}_{r}, t\right)=\hat{\mathbf{F}}\left(\hat{\mathbf{X}}_{\hat{r}}, t\right) \mathbf{H}^{T},
$$

for arbitrary orthogonal $\mathbf{H}$. Together, (5.40) and (5.41) yield the invariance condition

$$
\underline{\underline{f_{r}}}(\mathbf{F})=\underline{\underline{f_{r}}}(\mathbf{F} \mathbf{H})
$$

where $\mathbf{F}$ is an arbitrary deformation gradient tensor, and where $\mathbf{H}$ corresponds to an arbitrary orthogonal transformation.

This completes our general discussion of the restrictions which Postulate II places on all realistic measures of strain. The conclusion (5.42) is valid for simple strain measures. Again we remark that further results could be generated for the situation in which the neighborhood of influence $\mathfrak{R}(X)$ is considered large enough to include second and higher deformation gradients in the strain functional.

\section{Simple Strain}

For a simple strain measure we have shown in Sections 4 and 5 that at $(X, t)^{13}$,

where $\underline{f}$ satisfies

$$
\mathbf{D}(t)=\underline{\underline{f}}(\mathbf{F}(t))
$$

$$
\underline{\underline{f}}(\mathbf{Q}(t) \mathbf{F}(t))=\mathbf{Q}(t) \underline{\underline{f}}(\mathbf{F}(t)) \mathbf{Q}^{T}(t)
$$

for arbitrary proper orthogonal tensors $\mathbf{Q}(t)$, while

$$
\underline{\underline{f}}(\mathbf{F}(t))=\underline{\underline{f}}(\mathbf{F}(t) \mathbf{H})
$$

for arbitrary constant orthogonal tensors $\mathbf{H}$.

We shall return later to discuss the consequences of these functional equations. Rather, as a first objective in this section we show that a neces-

${ }^{13}$ We omit explicit mention of the material particle or reference configuration here and, whenever possible, in the remainder of this paper, as we shall now consider these two quantities fixed. It should be recalled, however, that in general the form of $f$ depends on the choice of reference configuration, that $\mathbf{F}$ depends on the position of the particle in its reference configuration, and that $\mathbf{D}$ is being evaluated at the particle in question. 
sary and sufficient condition for a strain measure to be "simple", is satisfaction of the implication

$$
\dot{\mathbf{F}}(t)=\mathbf{0} \Rightarrow \dot{\mathbf{D}}(t)=\mathbf{0} .
$$

Roughly, this states that the assumption of a finite non-zero neighborhood of influence in the definition of strain is inconsistent with the implication (6.4).

Clearly, if the strain measure is simple it follows from (6.1) that (6.4) is satisfied. Our object here is to show that the converse is also true. To this end, with fixed reference configuration $\mathrm{r}$ and particle $X$, choose a fixed time $t$ and associate with the arbitrary localization $\chi_{X}$ at 0 in $\mathfrak{N}_{r}(\mathbf{0})$ the constant tensor

$$
\mathbf{F}=\mathbf{F}(t)=\nabla \chi_{X}(\mathbf{0}, t),
$$

which is calculated as in (2.13). Now consider the homogeneous deformation whose localization at 0 in $\mathfrak{R}_{r}(0)$ is defined by

$$
\chi_{X^{+}}\left(\Delta \mathbf{Y}_{r}\right)=\mathbf{F} \Delta \mathbf{Y}_{r}, \quad \Delta \mathbf{Y}_{r} \in \mathfrak{N}_{r}(\mathbf{0}) .
$$

This localization has the property that

$$
\nabla \chi_{X^{+}}\left(\Delta \mathbf{Y}_{r}\right)=\nabla \chi_{X}(\mathbf{0}, t)=\mathbf{F} .
$$

In the present context, a theorem recently proved by GURTIN [12 $]^{14}$ shows that for fixed $\mathbf{r}$ and $X$, there exists a motion $\chi^{*}$ and a time $t^{*}$ whose localization satisfies the requirements

$$
\begin{aligned}
\chi_{X^{*}}\left(\Delta \mathbf{Y}_{r}, t^{*}\right) & =\chi_{X^{+}}\left(\Delta \mathbf{Y}_{r}\right), \\
\chi_{X^{*}}\left(\Delta \mathbf{Y}_{r}, t\right) & =\chi_{X}\left(\Delta \mathbf{Y}_{r}, t\right), \\
\nabla \dot{\boldsymbol{\chi}} x^{*}(\mathbf{0}, s) & =\mathbf{0} \text { at each time } s,
\end{aligned}
$$

for $\Delta \mathbf{Y}_{r} \in \mathfrak{R}_{r}(0)$. The strain associated with this localization is given at each time $s$ by the functional $f_{r}\left(\boldsymbol{\chi} x^{*}\left(\Delta \mathbf{Y}_{r}, s\right)\right)$ for $\Delta \mathbf{Y}_{r} \in \mathfrak{R}_{r}(\mathbf{0})$. In view of $(6.8 \mathrm{c})$, the conjecture $(6.4)$ implies that

$$
\frac{d}{d s} \underline{\underline{f}}\left(\boldsymbol{\chi} x^{*}\left(\Delta \mathbf{Y}_{r}, s\right)\right)=\mathbf{0} \text {. }
$$

Hence, by integration and application of $(6.8 \mathrm{a}, \mathrm{b})$ we obtain

$$
\underline{\underline{f_{r}}}\left(\boldsymbol{\chi}_{X} X^{+}\left(\Delta \mathbf{Y}_{r}\right)\right)=f_{\underline{r}}\left(\boldsymbol{\chi}_{X}\left(\Delta \mathbf{Y}_{r}, t\right)\right) .
$$

Finally, by defining the function $h_{r}$ thru

$$
\stackrel{h_{r}}{=}(\mathbf{A})=\underline{\underline{f_{r}}}\left(\mathbf{\Lambda} \Delta \mathbf{Y}_{r}\right), \quad \Delta \mathbf{Y}_{r} \in \Re_{r}(\mathbf{0}),
$$

for all constant second order tensors $\mathbf{A}$, we reach, with the aid of (6.6), $(6.10),(6.11)$, the result

$$
\left.\underline{f_{r}(\boldsymbol{\chi} X}\left(\Delta \mathbf{Y}_{r}, t\right)\right)=\underline{\underline{h_{r}}}(\mathbf{F}), \quad \Delta \mathbf{Y}_{r} \in \mathfrak{R}_{r}(\mathbf{0}) .
$$

14 See Lemma 2, p. 343. 
Thus, recalling that the left hand side of (6.12) represents the strain measure $\mathbf{D}(t)$ for fixed $X$, we are led to conclude that the strain measure must be simple, which completes the proof.

We turn now to discuss the functional equations (6.2), and (6.3). The solution to these equations has been given previously in several papers and treastise's on continuum mechanics $[1,2,3,4,5]$. Therefore, we merely remark that the polar decomposition ${ }^{15}$ of $\mathbf{F}(t)$, along with (6.1) and (6.3) yields the existence of a function $g$ such that

$$
\mathbf{D}(t)=\underline{\underline{g}}(\mathbf{B}(t)),
$$

where $\mathbf{B}(t)$, the left CaUCHY-GreEv strain tensor, is defined by

$$
\mathbf{B}(t)=\mathbf{F}(t) \mathbf{F}^{T}(t) .
$$

Moreover, (6.2) states that through $g$, the strain $\mathbf{D}(t)$ must be an isotropic tensor valued function of $\mathbf{B}(t)$,

$$
\underline{\underline{g}}\left(\mathbf{Q}(t) \mathbf{B}(t) \mathbf{Q}^{T}(t)\right)=\mathbf{Q}(t) \underline{\underline{g}}(\mathbf{B}(t)) \mathbf{Q}^{T}(t),
$$

and, therefore, admits the representation ${ }^{16}$

$$
\mathbf{D}(t)=\underline{\underline{g}}(\mathbf{B}(t))=\alpha_{0} \mathbf{1}+\alpha_{1} \mathbf{B}(t)+\alpha_{2} \mathbf{B}^{2}(t),
$$

where $\alpha_{i}(i=0,1,2)$ are scalar valued functions of the three principal invariants of $\mathbf{B}(t)$, defined by

$$
\begin{aligned}
I_{B} & =\operatorname{tr} \mathbf{B}, \\
I I_{B} & =\frac{1}{2}\left[(\operatorname{tr} \mathbf{B})^{2}-\operatorname{tr} \mathbf{B}^{2}\right], \\
I I I_{B} & =\operatorname{det} \mathbf{B} .
\end{aligned}
$$

Along with this representation we observe ${ }^{16}$ that for simple strain the strain tensor $\mathbf{D}(t)$ must be symmetric,

$$
\mathbf{D}(t)=\mathbf{D}^{T}(t) .
$$

Further, since $\mathbf{B}^{-1}(t)$ is an isotropic tensor valued function of $\mathbf{B}(t)$ and vice-versa, we could equally well have used $\mathbf{B}^{-1}(t)$ everywhere in place of $\mathbf{B}(t)$ above, without loss of generality.

KARNI and REInER [7] have called a strain measure $n^{\text {th }}$ order if it is an $n^{\text {th }}$ order polynomial in the displacement gradients. In this sense,

15 See, eg., Halmos $[11], \S 83$.

${ }_{16}$ The method used by SERRIN [6], §59, to deduce this representation does not. a priori require $g$ to be a symmetric function, but merely that its argument $\mathbf{B}$ be symmetric. The symmetry of $\mathbf{B}$ is obvious from (6.14).

We remark that although this representation is valid in all cases, there are examples of simple strain measures which are more conveniently left in their irrational. or trancendental forms. For example, see HENCKY's logarithmic measure discussed in TrUesdell [10], $\S 16,17$. Other examples and related remarks are given in [9], $\S \S 32,33$, along with a historical account of the theory of strain in $\S 33 \mathrm{~A}$. 
both $\mathbf{B}(t)$ and $\mathbf{B}^{-1}(t)$ are second order, as it follows from (6.14) and (2.19) that

$$
\mathbf{B}(t)=1+\mathbf{P}(t)+\mathbf{P}^{T}(t)+\mathbf{P}(t) \mathbf{P}^{T}(t),
$$

and from (6.14) and (2.20) that

$$
\mathbf{B}^{-1}(t)=\mathbf{1}-\mathbf{M}(t)-\mathbf{M}^{T}(t)+\mathbf{M}^{T}(t) \mathbf{M}(t) .
$$

Hence, from $(6.16)-(6.19)$ we see that there exists no property invariant first order measure of simple strain aside from a constant isotropic tensor ${ }^{17}$. Further, it follows that the most general properly invariant second order measure of simple strain must be of the form

$$
\mathbf{D}(t)=\left[\alpha_{00}+\alpha_{01} \operatorname{tr} \mathbf{B}(t)\right] \mathbf{1}+\alpha_{10} \mathbf{B}(t),
$$

where $\alpha_{00}, \alpha_{01}, \alpha_{10}$ are constants, and where for $\mathbf{B}(t)$ we may substitute (6.21). We remark that an expression similar to (6.23) may also be written where $\mathbf{B}(t)$ is replaced by $\mathbf{B}^{-1}(t)$ of $(6.22)$.

If a body is subjected to a rigid motion (relative to its reference configuration) it is usual to refer to the body as being unstrained (relative to its reference configuration). Neglecting the possibility of residual strain, this suggests the requirement that

$$
\mathbf{D}(t)=\mathbf{0} \text { if and only if } \chi \text { is rigid }^{18} \text {. }
$$

Due to the well known result ${ }^{19}$ that $\chi$ is rigid if and only if $\mathbf{B}(t)=\mathbf{1}$ for all $t$, it follows from (6.24) that

$$
\mathbf{D}(t)=\mathbf{0} \text { if and only if } \mathbf{B}(t)=1 \text {, for all } t .
$$

This condition rules out the possibility of a constant isotropic tensor as a first order simple strain measure, and implies the necessary and sufficient conditions

$$
\alpha_{10} \div 0, \quad \alpha_{00}+3 \alpha_{01}+\alpha_{10}=0,
$$

on the coefficients appearing in (6.23).

One of the most common second order simple strain measures corresponds to the choice $\alpha_{01}=0$ and $\alpha_{10}=\frac{1}{2}$. In this case,

$$
\mathbf{D}(t)=\frac{1}{2}(\mathbf{B}(t)-\mathbf{1})=\frac{1}{2}\left[\mathbf{P}(t)+\mathbf{P}^{T}(t)+\mathbf{P}(t) \mathbf{P}^{T}(t)\right] ;
$$

which Karni and ReIner [7] denote as the Green (final) strain tensor.

17 Only in the limit of infinitesimal deformation gradients is it possible to approximate the strain by a first order measure. This measure will not, however, be properly invariant.

is Here, $\boldsymbol{\chi}$ denotes the motion relative to the reference configuration as given in (2.3).

19 This follows from (6.14) and (2.12), and can be found in Mrcrar [8]. 
When $\mathbf{B}(t)$ is replaced by $\mathbf{B}^{-1}(t)$ in $(6.23)$, and we choose the corresponding $\alpha_{01}=0$ and $\alpha_{10}=-\frac{1}{2}$ then

$$
\mathbf{D}(t)=\frac{1}{2}\left(\mathbf{1}-\mathbf{B}^{-1}(t)\right)=\frac{1}{2}\left[\mathbf{M}(t)+\mathbf{M}^{T}(t)-\mathbf{M}^{T}(t) \mathbf{M}(t)\right],
$$

which is denoted as the Aumansi (final) strain tensor ${ }^{20}$ in the above referenced work of KARNI and REINER.

Finally, we remark that the analysis given here, and, in particular, the result (6.23), does not admit new second order spatial strain measures of the form proposed by KARNI and ReINER [7]; their strain measures are physically not acceptable due essentially to our Postulate I of observer invariance.

\section{Material Strain Measure}

All of the work in the preceeding sections of this paper was concerned with a spatial measure of strain, $\mathbf{D}(X, t)$. We could equally well have considered a material strain measure $\mathbf{D}_{r}(X, t)$ also defined through (3.1), (3.2), (3.3) but differing in interpretation from $\mathbf{D}(X, t)$ in the sense that in the same fixed coordinate system its components are calculated relative to base vectors at the position $\mathbf{X}_{r}$ of $X$ in reference configuration $\mathbf{r}$. Rather than formulate Postulates I, II and then reach independently all of the foregoing results in terms of $\mathbf{D}_{r}(X, t)$, it is sufficient to relate $\mathbf{D}_{r}(X, t)$ to $\mathbf{D}(X, t)$ by means of the motion relative to $\mathbf{r}$ given in (2.3). Then the results for $\mathbf{D}_{r}$ are immediate. The motion (2.3) acts as a change of coordinate system with regard to the tensor transformation $\mathbf{D}_{r} \leftrightarrow \mathbf{D}$. Hence, the deformation gradient tensor (2.12) serves as the transformation matrix and we have at $(X, t)$,

$$
\mathbf{D}=\mathbf{F} \mathbf{D}_{r} \mathbf{F}^{T} .
$$

With the relation (7.1), it is straightforward to transform all previous results of Sections 4,5 , and 6 from $\mathbf{D}$ to $\mathbf{D}_{r}$. We shall not dwell on this here, except to briefly mention some of the main results concerning simple strain considered in Section 6. From (6.16) and (7.1) we can construct the most general material measure of simple strain. Hence we have

$$
\mathbf{D}_{r}(t)=\mathbf{F}^{-1}(t)\left\{\alpha_{0} \mathbf{1}+\alpha_{1} \mathbf{B}(t)+\alpha_{2} \mathbf{B}^{2}(t)\right\}\left[\mathbf{F}^{T}(t)\right]^{-1} .
$$

which may be written as

$$
\mathbf{D}_{r}(t)=\beta_{0} \mathbf{1}+\beta_{\mathbf{1}} \mathbf{C}(t)+\beta_{\mathbf{2}} \mathbf{C}^{2}(t),
$$

where $\mathbf{C}(t)$, the right CAUCHY-GREeN strain tensor, is defined by

$$
\mathbf{C}(t)=\mathbf{F}^{T}(t) \mathbf{F}(t),
$$

and where $\beta_{i}(i=0,1,2)$ are scalar valued functions of the three principal invariants of $\mathbf{C}(t)$ (which, because of the forms of (6.14) and (7.4),

${ }^{20}$ See also Truesdell and ToupIN [9], $\S 31$, where this strain tensor is attributed to Ammansi and Hamex. 
are the same as the principal invariants of $\mathbf{B}(t)$ given in $(6.17)-(6.19))$. To obtain (7.3), substitute for $\mathbf{B}(t)$ in (7.2) the definition (6.14), use (7.4), and apply the Cayley-Hamilton equation

$$
I I I_{C} \mathbf{C}^{-1}=\mathbf{C}^{2}-I_{C} \mathbf{C}+I I_{C} \mathbf{1} .
$$

Hence, the most general material measure of simple strain must be an isotropic tensor valued function of $\mathbf{C}(t)$. Further, since $\mathbf{C}^{-1}(t)$ is an isotropic tensor valued function of $\mathrm{C}(t)$, and vice-versa, we can, without loss of generality, replace $\mathbf{C}(t)$ by $\mathbf{C}^{-1}(t)$ in $(7.3)$, where the sealar coefficients are understood to be different.

By (2.19), (2.20) and (7.4),

$$
\mathbf{C}(t)=\mathbf{1}+\mathbf{P}(t)+\mathbf{P}^{T}(t)+\mathbf{P}^{T}(t) \mathbf{P}(t),
$$

and

$$
\mathbf{C}^{-1}(t)=\mathbf{1}-\mathbf{M}(t)-\mathbf{M}^{T}(t)+\mathbf{M}(t) \mathbf{M}^{T}(t) .
$$

Hence, similar to the procedure used in Section 6 , we conclude that the most general properly invariant second order material measure of strain must be of the form

$$
\mathbf{D}_{r}(t)=\left[\beta_{00}+\beta_{0 \mathbf{1}} \operatorname{tr} \mathbf{C}(t)\right] \mathbf{1}+\beta_{\mathbf{1 0}} \mathbf{C}(t),
$$

where $\beta_{00}, \beta_{01}, \beta_{10}$ are constants. A similar equation holds for $\mathbf{C}(t)$ replaced by $\mathbf{C}^{-1}(t)$. Application of the restriction (6.24) results in the necessary and sufficient conditions

$$
\beta_{10} \neq 0 . \quad \beta_{00}+3 \beta_{01}+\beta_{10}=0 .
$$

The particular choice $\beta_{01}=0 \quad \beta_{10}=\frac{1}{2}$ yields the well known material measure of simple strain ${ }^{21}$

$$
\mathbf{D}_{r}(t)=\frac{1}{2}(\mathbf{C}(t)-\mathbf{1})=\frac{1}{2}\left[\mathbf{P}(t)+\mathbf{P}^{T}(t)+\mathbf{P}^{T}(t) \mathbf{P}(t)\right],
$$

designated as GREEN (initial) in the previously referenced work of KARNI and ReINer. When $\mathbf{C}(t)$ is replaced by $\mathbf{C}^{-1}(t)$ in (7.8), and we choose the analogous coefficients $\beta_{01}=0$ and $\beta_{10}=-\frac{1}{2}$, it follows that

$$
\mathbf{D}_{r}(t)=\frac{\mathbf{1}}{2}\left(\mathbf{1}-\mathbf{C}^{-1}(t)\right)=\frac{\mathbf{1}}{2}\left[\mathbf{M}(t)+\mathbf{M}^{T}(t)-\mathbf{M}(t) \mathbf{M}^{T}(t)\right],
$$

which corresponds to the Almanss (initial) strain tensor in the work of KARNI and ReINER.

Finally, we remark that (7.8) does not admit new second order material strain tensors of the form which were proposed by KARNI and REINER. This is due essentially to the fact that their proposed measures are not properly invariant.

21 Truesdell and Toupin [9], $\S 31$, attribute this strain tensor to Green and St. Venant. 


\title{
Acknowledgement
}

This research was supported by the National Science Foundation. We also gratefully acknowledge stimulating discussions with Dr. KARL ScHULER.

\section{References}

[1] Truesdelt, C. and W. Nolt: The Non-linear Field Theories of Mechanies. Flügge's Encyclopedia of Physics, Vol. III/3, Berlin: Springer, 1965.

[2] Nour, W.: A Mathematical Theory of the Mechanical Behavior of Continuous Media. Arch. Rat. Mech. Anal. 2, 197-226 (1958).

[3] Norc, W.: On the Continuity of Solid and Fluid States. J. Rat. Mech. Anal. 4, $3-81$ (1955).

[4] Rivin, R. S. and J. L. ERICksen: Stress-deformation Relations for Isotropic Materials. J. Rat. Mech. Anal. 4, 323-425 (1955).

[5] Ertagen, A. Cemal: Nonlinear Theory of Continuous Media. New York: McGraw-Hill, 1962.

[6] SERRIN, J.: Mathematical Principles of Classical Fluid Mechanics. Flügge's Encyclopedia of Physies, Vol. VIT/1, Berlin: Springer, 1959.

[7] Karni, Z. and M. Rerner: The General Measure of Deformation. Second Order Effects in Elasticity, Plasticity and Fluid Dynamics. Internat. Sympos. 217-227, Haifa, 1962.

[8] Mromal, A. D.: Matrix and Tensor Calculus. New York: John Wiley and Sons, Inc., 1947.

[9] Truesdeml, C. and R. Toupin: The Classical Field Theories. Flügge's Encyclopedia of Physics, Vol. ITI/1, Berlin: Springer, 1960.

[10] Truesderc, C.: The mechanical Foundations of Elasticity and Fluid Dynamics. J. Rat. Mech. Anal. 1, $125-300$ (1952).

[11] Harmos, Paur R.: Finite Dimensional Vector Spaces. Princeton, New Jersey: D. Van Nostrand Company, Inc., 1958.

[12] Gurtin, M. E.: Thermodynamics and the Possibility of Spatial Interaction in Elastic Materials. Arch. Rat. Mech. Anal. 19, 339-352 (1965).

\author{
Professor R. L. Fosdick \\ Department of Mechanics \\ Illinois Institute of Technology \\ Chicago, Illinois, U.S.A. \\ Professor A. S. Wineman \\ Department of Engineering Mechanics \\ University of Michigan \\ Ann Arbor, Michigan, U.S.A.
}

